\title{
Modelo de equilibrio químico para representar solubilidades de sistemas ternarios y su aplicación a la predicción de eutécticos de sistemas cuaternarios
}

\author{
Chemical equilibrium model to represent solubilities of ternary systems \\ and their application to the prediction of eutectic of quaternary systems
}

\author{
Jorge A. Lovera ${ }^{1} \quad$ Svetlana Ushak $^{2} \quad$ Elsa K. Flores $^{3}$ \\ Angel G. Fernández ${ }^{2} \quad$ Héctor Galleguillos ${ }^{2,3 *}$
}

Recibido 22 de marzo de 2017, aceptado 23 de diciembre de 2018

Received: March 22, 2017 Accepted: December 23, 2018

\begin{abstract}
RESUMEN
Incorporar la energía solar en el sector habitacional requiere de materiales adecuados para el almacenamiento de energía. Algunas mezclas eutécticas de sales inorgánicas hidratadas son una buena alternativa. Habitualmente métodos experimentales son empleados para encontrar una mezcla eutéctica con una temperatura de fusión próxima a las del medio ambiente. Un método alternativo, que no requiere de mucho tiempo y dinero, es predecir el punto eutéctico mediante un adecuado modelo termodinámico. En el presente estudio se ha realizado una rigurosa parametrización del modelo de Brunauer, Emmett y Teller (modelo BET) para representar exitosamente el equilibrio sólido-líquido (solubilidades) a 0 y $20^{\circ} \mathrm{C}$ de los sistemas ternarios $\mathrm{NaNO}_{3}+\mathrm{Ca}\left(\mathrm{NO}_{3}\right)_{2}+\mathrm{H}_{2} \mathrm{O}$ y $\mathrm{NH}_{4} \mathrm{NO}_{3}+\mathrm{Ca}\left(\mathrm{NO}_{3}\right)_{2}+\mathrm{H}_{2} \mathrm{O}$. El modelo de equilibrio químico obtenido se ha extendido para predecir los puntos eutécticos de dos sistemas cuaternarios: $\mathrm{LiNO}_{3}+\mathrm{NaNO}_{3}+\mathrm{Ca}\left(\mathrm{NO}_{3}\right)_{2}+\mathrm{H}_{2} \mathrm{O}$ y $\mathrm{LiNO}_{3}+\mathrm{NH}_{4} \mathrm{NO}_{3}+\mathrm{Ca}\left(\mathrm{NO}_{3}\right)_{2}+\mathrm{H}_{2} \mathrm{O}$. Las temperaturas de fusión predichas son $15,9^{\circ} \mathrm{C}$ y $3,9^{\circ} \mathrm{C}$, respectivamente. Conforme a estos resultados se puede concluir que el primer sistema cuaternario tiene un mayor potencial para ser usado como material para el almacenamiento de energía en edificios y hogares.
\end{abstract}

Palabras clave: Modelación termodinámica, predicción de punto eutéctico, mezcla acuosa de nitratos.

\begin{abstract}
Incorporating solar energy in the housing sector requires adequate materials for energy storage. Some eutectic mixtures of hydrated inorganic salts with near-ambient melting temperatures are a good alternative. Usually experimental methods are used to find a eutectic mixture with a fusion temperature close to that of the environment. An alternative method, which does not require much time and money, is to predict the eutectic point by means of an appropriate thermodynamic model. In the present study the BET model (Brunauer, Emmett and Teller model) was used to determine the eutectic points of two quaternary systems: $\mathrm{LiNO}_{3}+\mathrm{NaNO}_{3}+\mathrm{Ca}\left(\mathrm{NO}_{3}\right)_{2}+\mathrm{H}_{2} \mathrm{O}$ y $\mathrm{LiNO}_{3}+\mathrm{NH}_{4} \mathrm{NO}_{3}+\mathrm{Ca}\left(\mathrm{NO}_{3}\right)_{2}+\mathrm{H}_{2} \mathrm{O}$. The obtained chemical equilibrium model has been extended to predict the eutectic points of two quaternary systems: $\mathrm{LiNO}_{3}+\mathrm{NaNO}_{3}+\mathrm{Ca}\left(\mathrm{NO}_{3}\right)_{2}+\mathrm{H}_{2} \mathrm{O}$ and $\mathrm{LiNO} 3+\mathrm{NH}_{4} \mathrm{NO}_{3}+\mathrm{Ca}\left(\mathrm{NO}_{3}\right)_{2}+\mathrm{H}_{2} \mathrm{O}$. The predicted melting temperatures are $15.9^{\circ} \mathrm{C}$ and $3.9^{\circ} \mathrm{C}$, respectively. Based on these results, it can be concluded that the first quaternary system has a greater potential to be used as energy storage material in buildings and homes.
\end{abstract}

Keyword: Thermodynamic modeling, eutectic point prediction, aqueous nitrate mixture.

\footnotetext{
1 Facultad de Ingeniería y Arquitectura. Universidad Arturo Prat. Av. Arturo Prat 2120. Iquique, Chile.

2 Facultad de Ingeniería. Universidad de Antofagasta. Av. Angamos 601. Antofagasta, Chile.

3 CICITEM, Sucre No 220, Of. 620, Edificio Bulnes, Antofagasta, Chile.

* Autor de correspondencia: hector.galleguillos@uantof.cl
} 


\section{INTRODUCCIÓN}

El escenario energético mundial muestra que la energía consumida proviene en su mayor parte de fuentes no renovables, las cuales tiene una gran incidencia en la emisión de $\mathrm{CO}_{2}$ y el calentamiento global. Este escenario también muestra que una buena parte del consumo de energía es usada para operar sistemas de acondicionamiento de aire y calefacción residencial [1], por esta razón diversos estudios y aplicaciones tecnológicas en edificios están dirigidos a suplir este tipo de sistemas mediante el empleo de energía solar y materiales con capacidad de almacenamiento de calor, dotándolos con una mayor inercia térmica. Esto permite disminuir el consumo de combustibles fósiles y la emisión de $\mathrm{CO}_{2}$; además de promover el desarrollo de edificios más eficientes energéticamente.

El almacenamiento de energía térmica como calor latente es un buen método de almacenamiento cuando se dispone de una energía que es variable en el tiempo, tal como lo es la energía solar. Los materiales usados en este tipo de almacenamiento se denominan material de cambio de fase o bien mediante el acrónimo PCM (Phase Change Material). Estos materiales poseen valores altos de calor de fusión y por esta característica son capaces de almacenar o liberar grandes cantidades de energía en los procesos de fusión o solidificación [2]. Diversas sales inorgánicas hidratadas han sido investigadas como PCM para aplicarlas en el campo del almacenamiento de energía solar a bajas temperaturas, ya que sus temperaturas de fusión están entre 14 y $117^{\circ} \mathrm{C}$ y presentan capacidades de almacenamiento de calor latente volumétrico relativamente más altos que los compuestos orgánicos [3].

La aplicación del almacenamiento de energía en edificios requiere que los materiales de cambio de fase tengan puntos de fusión cercanos al intervalo de temperatura del medio ambiente, sin embargo pocas sales hidratadas cumplen con este requisito. Teóricamente, cuando dos o más sales hidratadas son mezcladas, un nuevo material eutéctico puede surgir, con punto de fusión cercano a las temperaturas medioambientales [4]. En muchos laboratorios de investigación se realizan mezclas de sales, variando sus porcentajes, hasta obtener una mezcla eutéctica con un punto de fusión adecuado para su aplicación en edificios. Sin embargo, este procedimiento consume tiempo y recursos económicos, por lo tanto el uso de un método predictivo, basado en termodinámica de electrolitos, resulta ser una buena alternativa. En la literatura científica se han informado algunos trabajos de esta naturaleza; generalmente en los trabajos de predicción se usa el modelo termodinámico de Brunauer, Emmett y Teller (modelo BET), modificado por Ally y Braunstein [5], ya que varias sales que forman parte de las mezclas analizadas son bastante solubles en medio acuoso.

Zeng y Voigt [6] usaron el modelo BET para el cálculo de solubilidades de algunos sistemas binarios y varios sistemas ternarios acuosos formado por la combinación de las siguientes sales: $\mathrm{LiNO}_{3}$, $\mathrm{NaNO}_{3}, \mathrm{Mg}\left(\mathrm{NO}_{3}\right)_{2}, \mathrm{Ca}\left(\mathrm{NO}_{3}\right)_{2}, \mathrm{Zn}\left(\mathrm{NO}_{3}\right)_{2}, \mathrm{LiCl}$, $\mathrm{CaCl}_{2}, \mathrm{LiClO}_{4}$, y $\mathrm{Ca}\left(\mathrm{ClO}_{4}\right)_{2}$. Los autores validaron sus resultados, fundamentalmente los relacionados con los sistemas ternarios, comparando los valores de solubilidad predichos por el modelo, con los obtenidos en forma experimental e informados en la literatura. Como consecuencia del buen acuerdo entre los valores predichos y los experimentales, los autores usaron también el modelo para predecir composiciones y temperaturas eutécticas de sistemas ternarios.

Una reciente versión del modelo BET, modificado por Abraham y Abraham [7], fue utilizado por Li et al. [8] para la predicción del eutéctico del sistema ternario $\mathrm{NH}_{4} \mathrm{NO}_{3}+\mathrm{LiNO}_{3}+\mathrm{H}_{2} \mathrm{O}$; la temperatura de fusión predicha fue verificado experimentalmente mediante el método T-History y la técnica DSC. En el modelo BET modificado por Ally y Braunstein [5] no se considera la interacción sal-sal en el sistema multicomponente, en cambio en la versión de Abraham y Abraham [7] esta interacción es tomada en cuenta. Cuando el mezclado de sales no es ideal las interacciones entre las sales son relevantes. De acuerdo a lo anterior Li et al. [8] consideró un parámetro de interacción entre las sales $\mathrm{NH}_{4} \mathrm{NO}_{3}-\mathrm{LiNO}_{3}$; esto le permitió alcanzar un mejor resultado predictivo y representar mejor el diagrama de fases de solubilidad del sistema ternario $\mathrm{NH}_{4} \mathrm{NO}_{3}$ $+\mathrm{LiNO}_{3}+\mathrm{H}_{2} \mathrm{O}$. En ese mismo trabajo los autores recalcularon los eutécticos de los sistemas ternarios $\mathrm{LiNO}_{3}+\mathrm{NaNO}_{3}+\mathrm{H}_{2} \mathrm{O}$ y $\mathrm{LiNO}_{3}+\mathrm{Mg}\left(\mathrm{NO}_{3}\right)_{2}+\mathrm{H}_{2} \mathrm{O}$ que habían sido predichos por Zeng y Voigt [6], además realizaron la predicción del eutéctico del sistema cuaternario $\mathrm{LiNO}_{3}+\mathrm{NaNO}_{3}+\mathrm{Mg}\left(\mathrm{NO}_{3}\right)_{2}+\mathrm{H}_{2} \mathrm{O}$. 
En el análisis de estos tres últimos sistemas no fue necesario considerar las interacciones salsal, pero el parámetro binario empleado para el $\mathrm{LiNO}_{3}$ fue de Zeng et al. [9] en vez de la referencia [6], esto debido a una mejor representación de solubilidades de los sistemas ternarios de litio con dicho parámetro. También estos autores verificaron experimentalmente los eutécticos predichos, observando un buen acuerdo entre los valores calculados y los experimentales [8]. Zeng et al. [10] modeló exitosamente los diagramas de solubilidad de nuevos sistemas ternarios del tipo $\mathrm{Mn}\left(\mathrm{NO}_{3}\right)_{2}+\mathrm{M}\left(\mathrm{NO}_{3}\right)_{\mathrm{n}}+\mathrm{H}_{2} \mathrm{O}(\mathrm{M}=\mathrm{Ca}, \mathrm{Mg}$ y Li), utilizando el modelo BET modificado. Se predijeron puntos eutécticos que podrían ser posibles materiales de cambio de fase; sin embargo los autores no verifican experimentalmente los eutécticos predichos.

Con el propósito de predecir puntos eutécticos de sistemas acuosos cuaternarios conteniendo nitratos, en el presente trabajo se ha predicho, en forma satisfactoria, actividad del agua a $25^{\circ} \mathrm{C}$ del sistema $\mathrm{NaNO}_{3}+\mathrm{Ca}\left(\mathrm{NO}_{3}\right)_{2}+\mathrm{H}_{2} \mathrm{O}$. Posteriormente se realizó una rigurosa parametrización del modelo BET para representar de manera exitosa la solubilidad de los sistemas ternarios $\mathrm{NaNO}_{3}+\mathrm{Ca}\left(\mathrm{NO}_{3}\right)_{2}+\mathrm{H}_{2} \mathrm{O}$ y $\mathrm{NH}_{4} \mathrm{NO}_{3}+\mathrm{Ca}\left(\mathrm{NO}_{3}\right)_{2}+\mathrm{H}_{2} \mathrm{O}$ a $0{ }^{\circ} \mathrm{C}$ y $20^{\circ} \mathrm{C}$. Los parámetros desarrollados en este trabajo, junto con otros informados en la literatura, permitió obtener un modelo de equilibrio químico coherente y confiable para extender su aplicación a la predicción de la composición y temperatura eutéctica de los sistemas cuaternarios $\mathrm{LiNO}_{3}+\mathrm{NaNO}_{3}+\mathrm{Ca}\left(\mathrm{NO}_{3}\right)_{2}+\mathrm{H}_{2} \mathrm{O}$ y $\mathrm{LiNO}_{3}+\mathrm{NH}_{4} \mathrm{NO}_{3}+\mathrm{Ca}\left(\mathrm{NO}_{3}\right)_{2}+\mathrm{H}_{2} \mathrm{O}$.

\section{MODELO TERMODINÁMICO}

El proceso de fusión de una sal hidratada $\left(\mathrm{Salt} \cdot \mathrm{nH}_{2} \mathrm{O}\right)$ a la temperatura absoluta $T$, puede ser representado convenientemente como una reacción química:

$$
\text { Salt } \cdot \mathrm{nH}_{2} \mathrm{O}(\mathrm{s})=\mathrm{Salt}(\mathrm{ac})+\mathrm{nH}_{2} \mathrm{O}(\mathrm{ac})
$$

donde $\mathrm{n}$ es el número de moléculas de agua y la fase líquida es una solución acuosa (ac) saturada de la sal. En la condición de equilibrio de fases el potencial químico $\mu$ de la sal hidratada en la fase sólida $(s)$ es igual al potencial químico de la sal en la fase líquida $(l)$, esto es: $\mu_{\text {Salt } \cdot n \mathrm{H}_{2} \mathrm{O}}^{s}=\mu_{\mathrm{Salt}^{\prime} \cdot n \mathrm{H}_{2} \mathrm{O} \text {. A partir }}^{l}$ de esta igualdad y desarrollando convenientemente se obtiene:

$$
k=a_{\text {Salt }} a_{w}^{n}
$$

En la ecuación (1) el término $k$ es la constante de equilibrio correspondiente a la reacción de disociación de la sal hidratada, $a_{\text {Salt }}$ y $a_{w}$ son respectivamente la actividad de la sal anhidra y del agua en la fase líquida. La constante de equilibrio depende de la temperatura, generalmente son informadas en la literatura como una correlación en función de la temperatura para varias sales inorgánicas, tanto anhidras como hidratadas. En la Tabla 1 se da esta constante termodinámica como una función de la temperatura para las fases sólidas que se pueden formar en los sistemas ternarios y cuaternarios abordados en el presente trabajo.

Ecuaciones del modelo BET modificado fueron formulados para sistemas multicomponentes y aplicados recientemente a sistemas ternarios y cuaternarios para el cálculo de la actividad de las sales y del agua $[5,6,8]$. Estas ecuaciones son:

$$
\begin{gathered}
\ln \left(a_{i}\right)=\ln \left(\frac{N_{i}}{\sum_{i} N_{i}}\right)+r_{i} \ln \left(1-\frac{N_{i(M)}}{r_{i} N_{i}}\right) \\
+\sum_{i \neq j} \frac{\Omega_{i j}}{R T} x_{j}^{2} \\
a_{w}=1-\frac{\sum_{i} N_{i(M)}}{N_{w}}
\end{gathered}
$$

Tabla 1. Constante de equilibrio para procesos de fusión de fases sólidas.

\begin{tabular}{|l|c|c|r|c|c|}
\hline \multicolumn{7}{|c|}{$\ln k=A+B / T+C \ln (T)$} \\
\hline \multicolumn{1}{|c|}{ Fase sólida } & A & B & C & Rango, $T / \mathbf{K}$ & Referencia \\
\hline $\mathrm{LiNO}_{3} \cdot 3 \mathrm{H}_{2} \mathrm{O}$ & $-148,57$ & 1330,03 & 23,915 & $273-301$ & {$[8]$} \\
\hline $\mathrm{NaNO}_{3}$ & 3,238 & $-1888,3$ & 0 & $273-292$ & {$[8]$} \\
\hline $\mathrm{NH}_{4} \mathrm{NO}_{3}$ & 52,450 & 3587,1 & $-7,3015$ & $293-368$ & {$[8]$} \\
\hline $\mathrm{Ca}\left(\mathrm{NO}_{3}\right)_{2} \cdot 4 \mathrm{H}_{2} \mathrm{O}$ & 6,263 & $\pm 5328,9$ & 0 & $283-316$ & {$[6]$} \\
\hline
\end{tabular}




$$
\frac{N_{i(M)} \sum_{i} N_{i(M)}}{\left(r_{i} N_{i}-N_{i(M)}\right)\left(N_{w}-\sum_{i} N_{i(M)}\right)}=\exp \left(-\frac{\Delta E_{i}}{R T}\right)
$$

Donde $a_{i}$ es la actividad de la sal anhidra $i, N_{i}$ y $N_{w}$ son el número de moles de la sal $i$ y del agua, $N_{i(M)}$ es el número de coordinación de agua asociada a la sal $i, x_{j}$ es la fracción molar de la sal $j$ en la mezcla anhidra, $r_{i}$ y $\Delta E_{i}$ (también denotado por $e_{i}$ ) son los parámetros binarios del modelo y son específicos para cada sal anhidra, y $\Omega_{i j}$ es el parámetro de interacción empírico entre las sales $i$-j $j$. Los parámetros binarios son conocidos y son dados como una correlación lineal con la temperatura para varias sales inorgánicas. En la Tabla 2 se dan los parámetros $r_{i}$ y $\Delta E_{i}\left(e_{i}\right)$ para las sales de los sistemas analizados en el presente estudio. El parámetro de mezcla $\Omega_{i j}$ son conocidos para algunas interacciones sal-sal y tienen una dependencia lineal con la temperatura. En la Tabla 3 se muestra el parámetro de mezcla $\Omega_{i j}$ para las interacciones $\mathrm{NH}_{4} \mathrm{NO}_{3}-\mathrm{LiNO}_{3}$ y $\mathrm{Ca}\left(\mathrm{NO}_{3}\right)_{2}-\mathrm{LiNO}_{3}$. Para los otros pares de interacciones sal-sal relacionados con las sales de los sistemas cuaternarios del presente trabajo, se desconocen las expresiones de $\Omega_{i j}$. Cuando el parámetro de mezcla $\Omega_{i j}$ es cero para todas las interacciones sal-sal, entonces se obtiene la versión del modelo BET de Ally y Braunstein [5].

\section{METODO DE CÁLCULO}

\section{Equilibrio líquido-vapor}

La ecuación de la actividad del agua, ecuación (3), junto con las ecuaciones de energía, ecuación (4), es combinado para la predicción de la actividad del agua en función de la concentración de los componentes salinos de una solución acuosa. Para un sistema ternario $\mathrm{Sal}(1)-\mathrm{Sal}(2)-\mathrm{H}_{2} \mathrm{O}$, la ecuación específica para la actividad del agua es:

$$
A_{w}=1-\frac{N_{1(M)}+N_{2(M)}}{N_{w}}
$$

Conocida la composición de una solución (o el número de moles de cada sal $N_{1}$ y $N_{2}$ ) se determina las variables $N_{1(\mathrm{M})}$ y $N_{2(\mathrm{M})}$ resolviendo las ecuaciones (4), donde el subíndice $i=1,2$. La solución se encuentra fácilmente aplicando algún método numérico como el de Newton-Raphson multivariable. Luego, los resultados obtenidos se sustituyen en la ecuación (5) para determinar $a_{w}$. Este procedimiento se repite para obtener nuevos valores de $a_{w}$ tantas veces como se varía el par $\left(N_{1}\right.$, $\mathrm{N}_{2}$ ). Obsérvese que la determinación de la actividad del agua no depende del parámetro de mezcla $\Omega_{i j}$, esto es porque dicho parámetro está presente solo en las expresiones matemáticas para las actividades de los componentes salinos.

\section{Solubilidades}

La ecuación de equilibrio sólido-líquido (1) y el modelo BET puede ser aplicado para la predicción de solubilidades de sales en soluciones acuosas multicomponentes. Para el sistema ternario definido de manera general en la sección anterior y a una determinada temperatura, la solubilidad de una sal es función de la concentración de la otra sal. Esto se muestra claramente si se sustituye en la ecuación de equilibrio, ecuación (1), las expresiones matemáticas de actividad de sal $a_{i}$ y del agua $a_{w}$

Tabla 2. Parámetros binarios del modelo BET.

\begin{tabular}{|l|c|c|c|c|c|}
\hline \multirow{2}{*}{ Sal } & \multicolumn{2}{|c|}{$r=a+b T$} & \multicolumn{2}{c|}{$e=c+d T(\mathbf{J} / \mathbf{m o l})$} & \multirow{2}{*}{ Fuente } \\
\cline { 2 - 5 } & a & b & c & d & \\
\hline $\mathrm{LiNO}_{3}$ & 2,766 & 0,000143 & $-6583,6$ & 5,495 & {$[8]$} \\
\hline $\mathrm{NaNO}_{3}$ & 1,8 & 0 & -1000 & 0 & {$[8]$} \\
\hline $\mathrm{NH}_{4} \mathrm{NO}_{3}$ & 1,63 & 0 & 890 & 0 & {$[8]$} \\
\hline $\mathrm{Ca}\left(\mathrm{NO}_{3}\right)_{2}$ & 5,032 & $-0,0042$ & -4183 & $-4,88$ & {$[6]$} \\
\hline
\end{tabular}

Tabla 3. Parámetro de mezcla para diferentes interacciones sal-sal.

\begin{tabular}{|l|c|c|c|c|}
\hline \multirow{2}{*}{ Sal-Sal } & \multicolumn{3}{|c|}{$\Omega=A+B T\left(\mathbf{J}^{*} \cdot \mathbf{m o l}^{-1}\right)$} & \multirow{2}{*}{ Fuente } \\
\cline { 2 - 4 } & $\mathbf{A}$ & $\mathbf{B}$ & Rango (K) & \\
\hline $\mathrm{NH}_{4} \mathrm{NO}_{3}-\mathrm{LiNO}_{3}$ & 2759,3 & $-14,286$ & $298-363$ & {$[8]$} \\
\hline $\mathrm{Ca}\left(\mathrm{NO}_{3}\right)_{2}-\mathrm{LiNO}_{3}$ & -5463 & 20 & $273-298$ & {$[11]$} \\
\hline
\end{tabular}


del modelo BET. Conforme a lo anterior, se puede escribir de manera funcional:

$$
F\left(T, w_{1}, w_{2}, N_{1(M)}, N_{2(M)}\right)=0
$$

Dado la temperatura absoluta $T$ y un valor para la fracción en peso de la sal $2\left(w_{2}\right)$, se resuelve simultáneamente la ecuación (6) y las ecuaciones de energía (4), mediante prueba y error (Newton-Raphson multivariable), para determinar la solubilidad de la sal 1 en fracción en peso $\left(w_{1}\right)$, y los números de coordinación $N_{1(\mathrm{M})}$ y $N_{2(\mathrm{M})}$. Sucesivamente se repite el proceso de cálculo para otros valores de $w_{2}$ a temperatura constante. El número de moles de cada sal, $N_{1}$ y $N_{2}$, están relacionados con las fracciones en peso, $w_{1} \mathrm{y}$ $w_{2}$, mediante la estequiometria. El procedimiento anterior es válido siempre y cuando los parámetros del modelo BET sean conocidos. En la situación de que los parámetros de mezcla $\Omega_{i j}$ sean desconocidos y necesarios para un cálculo de solubilidades más preciso, estos parámetros se pueden estimar ajustando los datos experimentales de solubilidad del sistema ternario al modelo mediante el método de mínimos cuadrados. Una vez conocido $\Omega_{i j}$, entonces se predice las solubilidades de las sales de acuerdo al procedimiento descrito en los párrafos anteriores.

\section{Mezclas eutécticas}

En este caso la ecuación de equilibrio sólido-líquido para un sistema cuaternario $\mathrm{Sal}(1)-\mathrm{Sal}(2)-\mathrm{Sal}(3)-$ $\mathrm{H}_{2} \mathrm{O}$ se escribe específicamente para cada fase sólida y combinando con las ecuaciones de actividad del modelo BET se obtiene un sistema de ecuaciones no lineales que se puede abreviar funcionalmente como:

$$
\begin{aligned}
& F_{i}\left(T, w_{1}, w_{2}, w_{3}, N_{1(M)}, N_{2(M)}, N_{3(M)}\right)=0 \\
& (i=1,2,3)
\end{aligned}
$$

El conjunto de ecuaciones (7) se resuelven simultáneamente con las ecuaciones de energía (4) por el método de Newton-Raphson multivarible para las incógnitas: $T, w_{1}, w_{2}, w_{3}, N_{1(M)}, N_{2(M)} \mathrm{y}$ $N_{3(M)}$. La fracción másica de agua en el sistema se determina por diferencia, es decir: $1-w_{1}-w_{2}-w_{3}$.

\section{RESULTADOS Y DISCUSIÓN}

\section{Equilibrio líquido-vapor}

Datos experimentales de la actividad del agua del sistema ternario $\mathrm{NaNO}_{3}+\mathrm{Ca}\left(\mathrm{NO}_{3}\right)_{2}+\mathrm{H}_{2} \mathrm{O}$ a $25^{\circ} \mathrm{C}$ fueron reportados por Kirgintsev y Lukyanov [12]. Por otro lado, el modelo BET fue utilizado para la predicción de la actividad del agua en función de la concentración molal de cada una de las sales (expresadas en moles de sal por kilogramo de agua). Los parámetros informados en Tabla 2 fueron usados en la predicción. En la Figura 1 se graficaron los resultados experimentales y en ella se ilustra la precisión del modelo BET. Obsérvese que cada línea representa una actividad del agua experimental constante. Sin embargo, el modelo predice para cada composición de una línea de actividad constante, valores de actividad moderadamente variable, por lo que se ha considerado un valor promedio. Este valor promedio se usó en la fórmula del porcentaje de error con el propósito de determinar la desviación entre los valores calculados y los experimentales. La ecuación del error se muestra en leyenda de Figura 1. Es sabido que usualmente la predicción del modelo BET es pobre cuando la actividad del agua es alta, esto es bien explicado en la referencia [13]. Para el sistema ternario en cuestión, la predicción de actividades del agua mediante el modelo BET tiene una precisión menor al 5\% en todos los casos analizados, lo cual es razonablemente aceptable para aplicaciones de ingeniería, a pesar de que las actividades son altas, están entre 0,8 y 0,9 .

\section{Solubilidades}

Las solubilidades de los sistemas ternarios $\mathrm{NaNO}_{3}+\mathrm{Ca}\left(\mathrm{NO}_{3}\right)_{2}+\mathrm{H}_{2} \mathrm{O}$ y $\mathrm{NH}_{4} \mathrm{NO}_{3}+\mathrm{Ca}\left(\mathrm{NO}_{3}\right)_{2}+\mathrm{H}_{2} \mathrm{O}$ fueron predichas con el modelo BET considerando las mezclas salinas como ideales, es decir cuando $\Omega_{i j}=0$. En las Figuras 2 y 3 se muestran los resultados calculados, los cuales se comparan con los datos

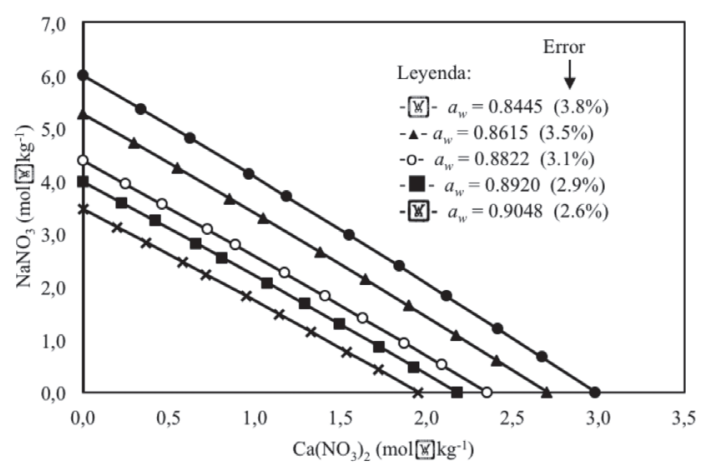

Figura 1. Actividad del agua experimental v/s molalidad del sistema ternario $\mathrm{NaNO}_{3}+\mathrm{Ca}\left(\mathrm{NO}_{3}\right)_{2}+\mathrm{H}_{2} \mathrm{O}$ y porcentaje de error del modelo BET (\%error $=\mid a_{w}$, $\left.\exp -\mathrm{a}_{\mathrm{w}, \text { cal }} \mid \mathrm{x} 100 / a_{w, \exp }\right)$. 
experimentales de Linke y Seidell [14]. Como se puede observar la representación de los datos experimentales con el modelo es inconsistente para ambos sistemas. Este desacuerdo de solubilidades para el sistema ternario $\mathrm{NaNO}_{3}+\mathrm{Ca}\left(\mathrm{NO}_{3}\right)_{2}+\mathrm{H}_{2} \mathrm{O}$ se puede atribuir a dos factores, primero debido a una inadecuada correlación de la constante de equilibrio del nitrato de sodio, la cual genera valores insuficientemente precisos a $0{ }^{\circ} \mathrm{C}$ y $20{ }^{\circ} \mathrm{C}$ (ver en Figura 2 la solubilidad del $\mathrm{NaNO}_{3}$ cuando la concentración de $\mathrm{Ca}\left(\mathrm{NO}_{3}\right)_{2}$ es cero), y segundo que la mezcla de sales es no ideal $\left(\Omega_{i j}{ }^{1} 0\right)$. En el caso del sistema ternario $\mathrm{NH}_{4} \mathrm{NO}_{3}+\mathrm{Ca}\left(\mathrm{NO}_{3}\right)_{2}+$ $\mathrm{H}_{2} \mathrm{O}$ las causas que explican la pobre predicción de solubilidades son: mezcla de sales no ideal y

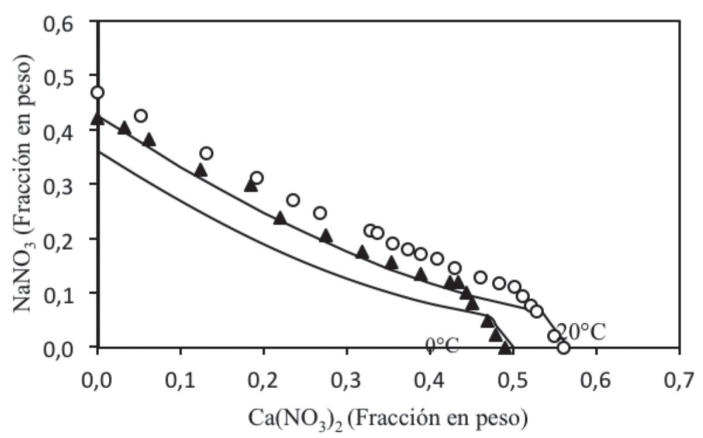

Figura 2. Solubilidad del sistema ternario $\mathrm{NaNO}_{3}+\mathrm{Ca}\left(\mathrm{NO}_{3}\right)_{2}+\mathrm{H}_{2} \mathrm{O}$. ( $\left.\boldsymbol{\Delta}\right),(\mathrm{O})$, Datos experimentales de Linke y Seidell [14]; (-), Calculado con el modelo BET considerando la mezcla de sales ideal $\left(\Omega_{\mathrm{ij}}=0\right)$.

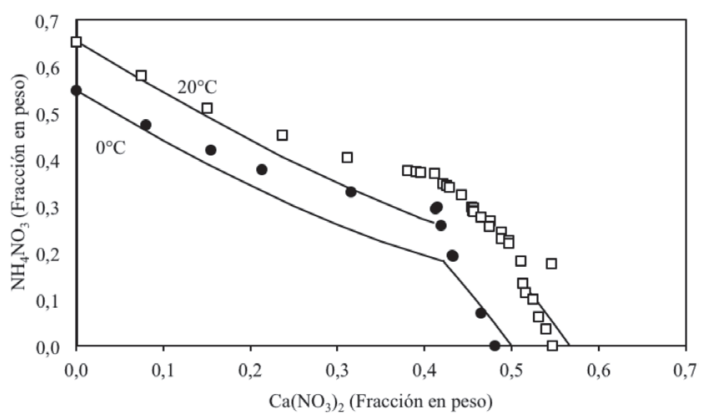

Figura 3. Solubilidad del sistema ternario $\mathrm{NH}_{4} \mathrm{NO}_{3}+\mathrm{Ca}\left(\mathrm{NO}_{3}\right)_{2}+\mathrm{H}_{2} \mathrm{O}$. ( $\left.\Delta\right)$, ( $)$, Datos experimentales de Linke y Seidell [14]; (-), Calculado con el modelo BET considerando la mezcla de sales ideal $\left(\Omega_{i j}=0\right)$. desconocimiento de las constantes de equilibrio de las reacciones de disociación para las sales dobles que se forman a $20^{\circ} \mathrm{C}\left(\mathrm{NH}_{4} \mathrm{NO}_{3} \times \mathrm{Ca}\left(\mathrm{NO}_{3}\right)_{2} \times 2 \mathrm{H}_{2} \mathrm{O}\right.$ y $\left.\mathrm{NH}_{4} \mathrm{NO}_{3} \times 5 \mathrm{Ca}\left(\mathrm{NO}_{3}\right)_{2} \times 10 \mathrm{H}_{2} \mathrm{O}\right)$.

Conforme a los resultados anteriores, se reajustó la constante de equilibro con datos experimentales de solubilidad [14] del sistema binario $\mathrm{NaNO}_{3}+\mathrm{H}_{2} \mathrm{O}$ en el rango de temperaturas de $0{ }^{\circ} \mathrm{C}$ a $100{ }^{\circ} \mathrm{C}$. $\mathrm{La}$ nueva correlación de equilibrio es:

$$
\ln k=-52.507+\frac{1120.0}{T}+8.0464 \ln T
$$

En la Figura 4 se muestra la calidad del ajuste en términos de la fracción molar del agua en función de la temperatura absoluta. Se graficaron en estas coordenadas para poder reproducir parte de los resultados de Ally [15] y compararlo con los resultados obtenidos al usar la nueva correlación para $k$. Ally en su estudio de la predicción de la curva de líquido para el sistema $\mathrm{NaNO}_{3}+\mathrm{H}_{2} \mathrm{O}$ utilizó la correlación de $k$ informada por Li et al. [8] y que aparece recopilada en la Tabla 1. Como se puede observar la predicción de solubilidad de Ally, representada por la línea discontinua, es pobre cerca de $273 \mathrm{~K}\left(0{ }^{\circ} \mathrm{C}\right)$. Es evidente que el modelo BET con la nueva correlación para $k$, ecuación (8), da una mejor representación de solubilidad en el rango de $0{ }^{\circ} \mathrm{C}$ a $100{ }^{\circ} \mathrm{C}$.

El parámetro de mezcla $\Omega_{i j}$ se estimó a $0{ }^{\circ} \mathrm{C}$ y $20^{\circ} \mathrm{C}$ con datos experimentales de solubilidad para cada sistema ternario $\left(\mathrm{NaNO}_{3}+\mathrm{Ca}\left(\mathrm{NO}_{3}\right)_{2}+\mathrm{H}_{2} \mathrm{O}\right.$ y $\left.\mathrm{NH}_{4} \mathrm{NO}_{3}+\mathrm{Ca}\left(\mathrm{NO}_{3}\right)_{2}-\mathrm{H}_{2} \mathrm{O}\right)$. En la Tabla 4 se

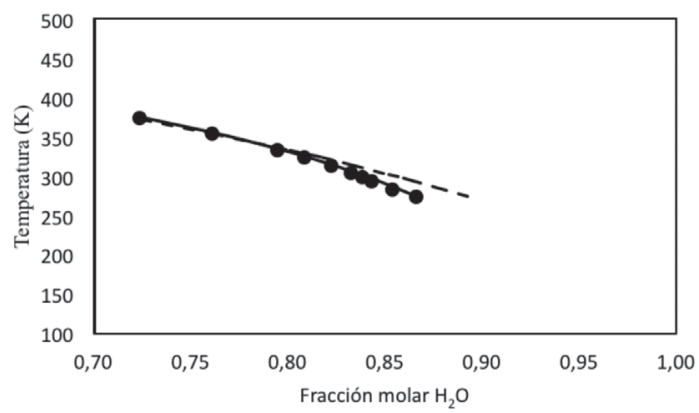

Figura 4. Solubilidad del sistema binario $\mathrm{NaNO}_{3}+\mathrm{H}_{2} \mathrm{O} .(\cdot)$, Dato experimental de Linke y Seidell [14]; (--), Dato calculado con el modelo BET y la correlación de $\mathrm{k}$ de Li et al. [8]; (-), Dato calculado con el modelo BET y la nueva correlación de $\mathrm{k}=-52,507+1120 / T+8,0464 \ln T$. 
dan los valores óptimos de este parámetro. El parámetro de mezcla que cuantifica la interacción $\mathrm{NaNO}_{3}-\mathrm{Ca}\left(\mathrm{NO}_{3}\right)_{2}$ resultó de tipo simétrico $\left(\Omega_{i j}=\Omega_{j i}\right)$, mientras que para las interacciones $\mathrm{NH}_{4} \mathrm{NO}_{3}-\mathrm{Ca}\left(\mathrm{NO}_{3}\right)_{2}$ fue de tipo asimétrico $\left(\Omega_{i j}\right.$ ${ }^{1} \Omega_{j i}$ ). Para los datos de solubilidad a $20{ }^{\circ} \mathrm{C}$ del segundo sistema ternario fue necesario estimar los parámetros y las constantes de equilibrio de las sales dobles $\mathrm{NH}_{4} \mathrm{NO}_{3} \times \mathrm{Ca}\left(\mathrm{NO}_{3}\right)_{2} \times 2 \mathrm{H}_{2} \mathrm{O}$ y $\mathrm{NH}_{4} \mathrm{NO}_{3} \times 5 \mathrm{Ca}\left(\mathrm{NO}_{3}\right)_{2} \times 10 \mathrm{H}_{2} \mathrm{O}$ (se informan al pie de Tabla 4). Con los parámetros del modelo BET informados en Tablas 1-3, salvo lnk del $\mathrm{NaNO}_{3}$, más la parametrización anterior, se calculó nuevamente las solubilidades de los sistemas ternarios $\mathrm{NaNO}_{3}+\mathrm{Ca}\left(\mathrm{NO}_{3}\right)_{2}+\mathrm{H}_{2} \mathrm{O}$ y $\mathrm{NH}_{4} \mathrm{NO}_{3}+$ $\mathrm{Ca}\left(\mathrm{NO}_{3}\right)_{2}+\mathrm{H}_{2} \mathrm{O}$. Los nuevos resultados se presentan en las Figuras 5 y 6, los cuales muestran que ahora

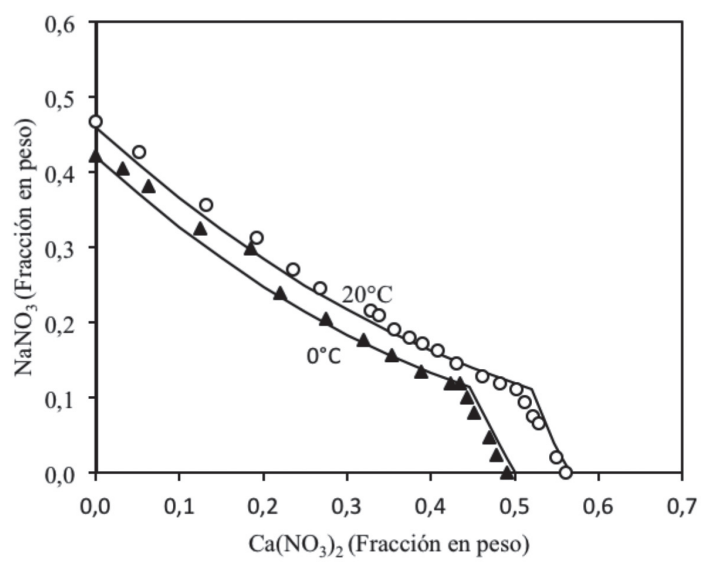

Figura 5. Solubilidad del sistema ternario $\mathrm{NaNO}_{3}+\mathrm{Ca}\left(\mathrm{NO}_{3}\right)_{2}+\mathrm{H}_{2} \mathrm{O}$. ( $\left.\boldsymbol{\Delta}\right),(\mathrm{o})$, Datos experimentales de Linke y Seidell [14]; (-), Calculado con el modelo BET considerando la mezcla de sales no ideal $\left(\Omega_{i j}{ }^{1} 0\right)$.

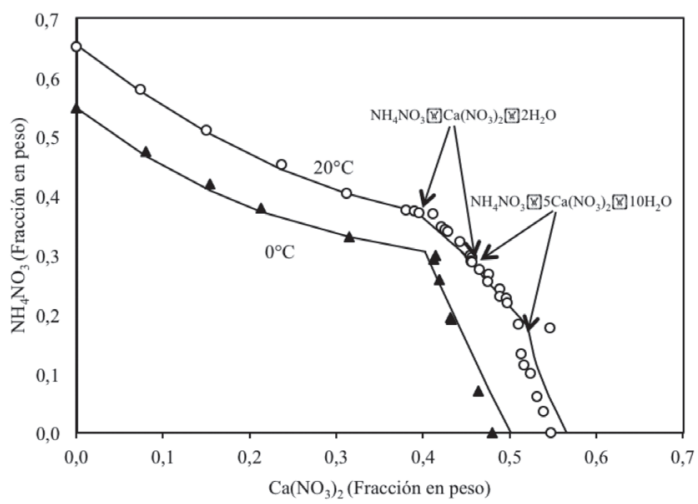

Figura 6. Solubilidad del sistema ternario $\mathrm{NH}_{4}$ $\mathrm{NO}_{3}+\mathrm{Ca}\left(\mathrm{NO}_{3}\right)_{2}+\mathrm{H}_{2} \mathrm{O}$. ( $\left.\boldsymbol{\Delta}\right)$, ( ( ), Datos experimentales de Linke y Seidell [14]; (-), Calculado con el modelo BET considerando la mezcla de sales no ideal $\left(\Omega_{i j}{ }^{1} 0\right)$.

sí los valores calculados son consistentes con los datos experimentales [14]. Por consiguiente queda comprobado, en forma exitosa, la capacidad del modelo de equilibrio químico desarrollado en este trabajo para la predicción de solubilidades de los sistemas ternarios analizados.

\section{Mezclas eutécticas}

Con el trabajo desarrollado en apartado anterior se ha logrado una parametrización rigurosa del modelo termodinámico BET, permitiéndonos obtener un modelo de equilibrio químico coherente y confiable para predecir solubilidades de sistemas ternarios; por esta razón se espera que la extensión del modelo a sistemas cuaternarios también pueda ser confiable. Este modelo químico, junto con las ecuaciones del proceso de fusión de fases sólidas, se utilizó para predecir mezclas eutécticas de los sistemas cuaternarios $\mathrm{LiNO}_{3}(1)+\mathrm{NaNO}_{3}(2$

Tabla 4. Parámetros de mezcla óptimos del modelo BET y constantes de equilibrio.*

\begin{tabular}{|c|c|c|c|}
\hline Sistema ternario & $t\left({ }^{\mathbf{}} \mathbf{C}\right)$ & $\Omega_{i j}\left(\mathbf{J} \times \mathbf{m o l}^{-\mathbf{1}}\right)$ & $\Omega_{j i}\left(\mathbf{J} \times \mathbf{m o l} \mathbf{l}^{-\mathbf{1}}\right)$ \\
\hline \multirow{2}{*}{$\mathrm{NaNO}_{3}-\mathrm{Ca}\left(\mathrm{NO}_{3}\right)_{2}-\mathrm{H}_{2} \mathrm{O}$} & 0 & $-1636,65$ & $-1636,65$ \\
\cline { 2 - 4 } & 20 & $-1985,96$ & $-1985,96$ \\
\hline \multirow{2}{*}{$\mathrm{NH}_{4} \mathrm{NO}_{3}-\mathrm{Ca}\left(\mathrm{NO}_{3}\right)_{2}-\mathrm{H}_{2} \mathrm{O}$} & 0 & $-8492,98$ & $-2390,93$ \\
\cline { 2 - 4 } & 20 & $-8824,17$ & $-2350,05$ \\
\hline
\end{tabular}

* Fase sólida: $\mathrm{NH}_{4} \mathrm{NO}_{3} \times \mathrm{Ca}\left(\mathrm{NO}_{3}\right)_{2} \times 2 \mathrm{H}_{2} \mathrm{O}$ y $\ln k=-11,506$ a $20^{\circ} \mathrm{C}$;

Fase sólida: $\mathrm{NH}_{4} \mathrm{NO}_{3} \times 5 \mathrm{Ca}\left(\mathrm{NO}_{3}\right)_{2} \times 10 \mathrm{H}_{2} \mathrm{O}$ y $\ln k=-51,279$ a $20^{\circ} \mathrm{C}$. 
)$+\mathrm{Ca}\left(\mathrm{NO}_{3}\right)_{2}(3)+\mathrm{H}_{2} \mathrm{O}$ y $\mathrm{LiNO}_{3}(1)+\mathrm{NH}_{4} \mathrm{NO}_{3}(2)$ $+\mathrm{Ca}\left(\mathrm{NO}_{3}\right)_{2}(3)+\mathrm{H}_{2} \mathrm{O}$. El método de predicción está descrito en una de las secciones de Método de Cálculo. Los parámetros de las Tablas 1 a 3 fueron considerados en dicha predicción, salvo la constante de equilibrio del $\mathrm{NaNO}_{3}$, donde se usó la recalculada en este trabajo. Previamente se encontró los coeficientes de la ecuación $\Omega=A+$ $B \times T$ para las interacciones $\mathrm{NaNO}_{3}-\mathrm{Ca}\left(\mathrm{NO}_{3}\right)_{2}$ y $\mathrm{NH}_{4} \mathrm{NO}_{3}-\mathrm{Ca}\left(\mathrm{NO}_{3}\right)_{2}$ con información de la Tabla 4; estos coeficientes se muestran en la Tabla 5. Los resultados de temperatura y composición de las mezclas eutécticas se presentan en la Tabla 6 para dos casos: 1) cuando las interacciones $\mathrm{NaNO}_{3}-\mathrm{Ca}\left(\mathrm{NO}_{3}\right)_{2}$ y $\mathrm{NH}_{4} \mathrm{NO}_{3}-\mathrm{Ca}\left(\mathrm{NO}_{3}\right)_{2}$ son ignoradas $\left(\Omega_{23}=0\right)$ y 2) cuando las interacciones $\mathrm{NaNO}_{3}-\mathrm{Ca}\left(\mathrm{NO}_{3}\right)_{2}$ y $\mathrm{NH}_{4} \mathrm{NO}_{3}-\mathrm{Ca}\left(\mathrm{NO}_{3}\right)_{2}$ son consideradas $\left(\Omega_{23}{ }^{1} 0\right)$.

Tabla 5. Coeficientes de la correlación del parámetro de mezcla con la temperatura.

\begin{tabular}{|l|c|c|}
\hline \multicolumn{3}{|c|}{$\Omega=A+B \times T\left(\mathbf{J} \cdot \mathbf{m o l}^{-\mathbf{1}}\right)$} \\
\hline Sal-Sal & A & B \\
\hline $\mathrm{NaNO}_{3}-\mathrm{Ca}\left(\mathrm{NO}_{3}\right)_{2}$ & 3134,1 & $-17,466$ \\
\hline $\mathrm{NH}_{4} \mathrm{NO}_{3}-\mathrm{Ca}\left(\mathrm{NO}_{3}\right)_{2}$ & $-3969,8$ & $-16,560$ \\
\hline $\mathrm{Ca}\left(\mathrm{NO}_{3}\right)_{2}-\mathrm{NH}_{4} \mathrm{NO}_{3}$ & $-2949,2$ & 2,0440 \\
\hline
\end{tabular}

De acuerdo a los resultados de la Tabla 6 , se puede deducir que el efecto de las interacciones $\mathrm{NaNO}_{3}-$ $\mathrm{Ca}\left(\mathrm{NO}_{3}\right)_{2}$ en la predicción del eutéctico del sistema cuaternario $\mathrm{LiNO}_{3}+\mathrm{NaNO}_{3}+\mathrm{Ca}\left(\mathrm{NO}_{3}\right)_{2}+\mathrm{H}_{2} \mathrm{O}$ es levemente significativo, como puede apreciarse en las pequeñas diferencias de temperatura y composición entre una y otra alternativa. En el caso del otro sistema cuaternario $\mathrm{LiNO}_{3}+\mathrm{NH}_{4} \mathrm{NO}_{3}+\mathrm{Ca}\left(\mathrm{NO}_{3}\right)_{2}+\mathrm{H}_{2} \mathrm{O}$, el efecto de las interacciones $\mathrm{NH}_{4} \mathrm{NO}_{3}-\mathrm{Ca}\left(\mathrm{NO}_{3}\right)_{2}$ es altamente significativo debido a las diferencias de los resultados de temperatura y composición obtenidos con y sin parámetros de mezcla. El eutéctico calculado con $\Omega_{23} \neq 0$ es más confiable.
Además se pueden obtener otros eutécticos para el sistema que contiene $\mathrm{NH}_{4} \mathrm{NO}_{3}$ en la medida que se consideren en el proceso de fusión de la mezcla salina otras fases sólidas, como por ejemplo las sales dobles $\left(\mathrm{NH}_{4} \mathrm{NO}_{3} \times \mathrm{Ca}\left(\mathrm{NO}_{3}\right)_{2} \times 2 \mathrm{H}_{2} \mathrm{O}\right.$ y $\left.\mathrm{NH}_{4} \mathrm{NO}_{3} \times 5 \mathrm{Ca}\left(\mathrm{NO}_{3}\right)_{2} \times 10 \mathrm{H}_{2} \mathrm{O}\right)$. Sin embargo, esto requiere contar con más información a diferentes temperaturas de datos de solubilidad del sistema ternario $\mathrm{NH}_{4} \mathrm{NO}_{3}+\mathrm{Ca}\left(\mathrm{NO}_{3}\right)_{2}+\mathrm{H}_{2} \mathrm{O}$, puesto que como se vio en cálculo de solubilidades de sección anterior, el $\mathrm{NH}_{4} \mathrm{NO}_{3}$ tiene tendencia a asociarse con el $\mathrm{Ca}\left(\mathrm{NO}_{3}\right)_{2}$ y el $\mathrm{H}_{2} \mathrm{O}$ para formar sales dobles hidratadas. El argumento anterior es concordante con el hecho de que la predicción del eutéctico del sistema cuaternario conteniendo $\mathrm{NH}_{4} \mathrm{NO}_{3}$ ha sido más complejo que el sistema que contiene $\mathrm{NaNO}_{3}$, puesto que la presencia del nitrato de amonio ha exigido la necesidad de caracterizar el parámetro de mezcla de tipo asimétrico, es decir $\Omega_{23} \neq \Omega_{32}$.

En relación a las temperaturas de los puntos eutécticos de los dos sistemas cuaternarios analizados en este trabajo, el sistema $\mathrm{LiNO}_{3}-\mathrm{NaNO}_{3}+\mathrm{Ca}\left(\mathrm{NO}_{3}\right)_{2}+\mathrm{H}_{2} \mathrm{O}$ tiene un punto de fusión $\left(\approx 16^{\circ} \mathrm{C}\right)$ que está próximo al intervalo de temperaturas del confort ambiental $\left(18-24{ }^{\circ} \mathrm{C}\right)$, razón por lo cual presenta un buen potencial como material para el almacenar energía en edificios y hogares. En todo caso es necesario realizar estudios experimentales más extensos para garantizar y completar la caracterización de este potencial PCM.

\section{CONCLUSIONES}

La actividad del agua en soluciones acuosas de $\mathrm{NaNO}_{3}+\mathrm{Ca}\left(\mathrm{NO}_{3}\right)_{2}+\mathrm{H}_{2} \mathrm{O}$ ha sido predicha con el modelo de BET. La predicción muestra una coincidencia razonablemente aceptable cuando se comparan con los datos experimentales de la literatura. La mayor desviación de los valores predichos fue de $3,9 \%$, menor al $5 \%$, por lo cual el modelo puede ser útil para aplicaciones de ingeniería.

Tabla 6. Temperatura y composición (\% en peso) de mezclas eutécticas predichas.

\begin{tabular}{|c|c|c|c|c|c|c|}
\hline Sistema cuaternario & Variante & $t\left({ }^{\circ} \mathbf{C}\right)$ & $\boldsymbol{\%}_{\mathbf{L i N O}} \mathbf{3}_{\mathbf{3}} \times \mathbf{3 H}_{\mathbf{2}} \mathbf{O}$ & $\mathbf{\%} \mathbf{N a N O}_{\mathbf{3}}$ & $\boldsymbol{\%}_{\mathbf{N H}} \mathbf{N}_{\mathbf{4}} \mathbf{N O}_{\mathbf{3}}$ & $\mathbf{\%} \mathbf{C a}\left(\mathbf{N O}_{\mathbf{3}}\right)_{\mathbf{2}} \times \mathbf{4} \mathbf{H}_{\mathbf{2}} \mathbf{O}$ \\
\hline $\mathrm{LiNO}_{3}-\mathrm{NaNO}_{3}-$ & $\Omega_{23}=0$ & 16,3 & 39,47 & 5,49 & & 55,04 \\
\cline { 2 - 7 } $\mathrm{Ca}\left(\mathrm{NO}_{3}\right)_{2}-\mathrm{H}_{2} \mathrm{O}$ & $\Omega_{23}{ }^{1} 0$ & 15,9 & 38,89 & 6,09 & & 55,02 \\
\hline $\mathrm{LiNO}_{3}-\mathrm{NH}_{4} \mathrm{NO}_{3}-$ & $\Omega_{23}=0$ & 8,0 & 33,83 & & 19,45 & 46,72 \\
\cline { 2 - 7 } $\left.\mathrm{Ca}_{23} \mathrm{NO}_{3}\right)_{2}-\mathrm{H}_{2} \mathrm{O}$ & $\Omega_{23} 0$ & 3,9 & 29,31 & & 23,23 & 47,46 \\
\hline
\end{tabular}


Las solubilidades de sales de nitrato, asociados a los equilibrio sólido-líquido de los sistemas ternarios $\mathrm{NaNO}_{3}+\mathrm{Ca}\left(\mathrm{NO}_{3}\right)_{2}+\mathrm{H}_{2} \mathrm{O}$ y $\mathrm{NH}_{4} \mathrm{NO}_{3}+\mathrm{Ca}\left(\mathrm{NO}_{3}\right)_{2}-\mathrm{H}_{2} \mathrm{O}$ a $0{ }^{\circ} \mathrm{C}$ y $20{ }^{\circ} \mathrm{C}$, son representadas exitosamente con el modelo BET. Para llevar a cabo esto fue necesario hacer una parametrización rigurosa del modelo BET. En este caso, además de emplear los parámetros del modelo BET informado en la literatura, fue pertinente estimar los parámetros de mezcla $\Omega_{i j}$ para ambos sistemas, además de una re-parametrización de la ecuación de la constante de equilibrio para la sal $\mathrm{NaNO}_{3}$ y la estimación de las constantes de equilibrio a $20^{\circ} \mathrm{C}$ para las fases sólidas $\mathrm{NH}_{4} \mathrm{NO}_{3} \times \mathrm{Ca}\left(\mathrm{NO}_{3}\right)_{2} \times 2 \mathrm{H}_{2} \mathrm{O}$ y $\mathrm{NH}_{4} \mathrm{NO}_{3} \times 5 \mathrm{Ca}\left(\mathrm{NO}_{3}\right)_{2} \times 10 \mathrm{H}_{2} \mathrm{O}$.

El modelo de equilibrio químico para representar solubilidades de los dos sistemas ternarios se extendió a la predicción de los puntos eutécticos de los sistemas $\mathrm{LiNO}_{3}-\mathrm{NaNO}_{3}+\mathrm{Ca}\left(\mathrm{NO}_{3}\right)_{2}+\mathrm{H}_{2} \mathrm{O}$ y $\mathrm{LiNO}_{3}+\mathrm{NH}_{4} \mathrm{NO}_{3}+\mathrm{Ca}\left(\mathrm{NO}_{3}\right)_{2}+\mathrm{H}_{2} \mathrm{O}$. En la pre-dicción se observa que el parámetro de mezcla que cuantifica la interacción $\mathrm{NH}_{4} \mathrm{NO}_{3}-\mathrm{Ca}\left(\mathrm{NO}_{3}\right)_{2}$ es mucho más significativo que la interacción $\mathrm{NaNO}_{3}-\mathrm{Ca}\left(\mathrm{NO}_{3}\right)_{2}$. Las temperaturas eutécticas calculadas del primer y segundo sistema cuaternario son $15,9^{\circ} \mathrm{C}$ y $3,9^{\circ} \mathrm{C}$, respectivamente. Conforme a estas temperaturas se concluye que el primer sistema cuaternario presenta un mejor potencial para almacenar energía en el sector habitacional.

\section{AGRADECIMIENTOS}

Los autores agradecen a CONICYT, a través del Proyecto FONDAP 15110019 (SERC-Chile), y a la VRIIP de la Universidad Arturo Prat mediante el Proyecto VRIIP0084-15.

\section{REFERENCIAS}

[1] S. Guichard, F. Miranville, D. Bigot and H. Boyer. "A thermal model for phase change materials in a building roof for a tropical and humid climate: Model description and elements of validation". Energy and Buildings, Vol. 70, pp. 71-80, 2014.

[2] S.D. Sharma and K. Sagara. "Latent heat storage materials and systems: a review". International Journal of Green Energy, Vol. 2, pp. 1-56, 2005.

[3] A. Sharma, V.V. Tyagi, C.R. Chen and D. Buddhi. "Review on thermal energy storage with phase change materials and applications". Renewable and Sustainable Energy Reviews. Vol. 13, pp. 318-345, 2009.

[4] W. Voigt and D. Zeng. "Solid-liquid equilibria in mixtures of molten salt hydrates for the design of heat storage materials". Pure and Applied Chemistry. Vol. 74, pp. 1909-1920, 2002.

[5] M.R. Ally and J. Braunstein. "Statistical mechanics of multilayer adsorption: electrolyte and water activities in concentrated solutions". J. Chem. Thermodyn. Vol. 30, pp. 49-58, 1998.

[6] D. Zeng and W. Voigt. "Phase diagram calculation of molten salt hydrates using the modified BET equation". CALPHAD. Vol. 27, pp. 243-251, 2003.

[7] M.Abraham and M.C.Abraham. "Electrolyte and water activities in very concentrated solutions". Electrochim. Acta. Vol. 46, 137-142, 2000.

[8] B. Li, D. Zeng, X. Yin and Q. Chen. "Theoretical prediction and experimental determination of room-temperature phase change materials using hydrated salts as agents". J. Therm. Anal. Calorim. Vol. 100, pp. 685-693, 2010.

[9] D. Zeng, J. Ming and W. Voigt. "Thermodynamic study of the system $(\mathrm{LiCl}+$ LiNO3+H2O)". J. Chem. Thermodynamics Vol. 40, pp. 232-239, 2008.

[10] D. Zeng, S. Fan and S. Chen. "Phase diagram prediction of systems $\mathrm{Mn}\left(\mathrm{NO}_{3}\right)_{2}-\mathrm{M}\left(\mathrm{NO}_{3}\right)$ $\mathrm{n}-\mathrm{H}_{2} \mathrm{O}(\mathrm{M}=\mathrm{Ca}, \mathrm{Mg}$ and $\mathrm{Li})$ with BETmodel". Trans. Nonferrous Met. Soc. China. Vol. 14, pp. 1192-1198, 2004.

[11] J. Jiang, X. Yin, D. Li, Y. Tan and D. Zeng. "Solubility phase diagram of the $\mathrm{Ca}\left(\mathrm{NO}_{3}\right)_{2}-$ $\mathrm{LiNO}_{3}-\mathrm{H}_{2} \mathrm{O}$ system". Journal of Chemical \& Engineering Data. Vol. 61, pp. 1125-1130, 2016.

[12] A.N. Kirgintsev and A.V. Lukyanov. "Isopiestic investigation of ternary solutions V. Ternary $\mathrm{NaNO}_{3}-\mathrm{Ca}\left(\mathrm{NO}_{3}\right)_{2}-\mathrm{H}_{2} \mathrm{O}, \mathrm{NaNO}_{3}-$ $\mathrm{La}\left(\mathrm{NO}_{3}\right)_{2}-\mathrm{H}_{2} \mathrm{O}, \mathrm{NaNO}_{3}-\mathrm{Th}\left(\mathrm{NO}_{3}\right)_{2}-\mathrm{H}_{2} \mathrm{O}$, $\mathrm{NaCl}-\mathrm{CaCl}_{2}-\mathrm{H}_{2} \mathrm{O}, \mathrm{NaCl}-\mathrm{LaCl}_{3}-\mathrm{H}_{2} \mathrm{O}$ and $\mathrm{NaCl}-\mathrm{ThCl}_{4}-\mathrm{H}_{2} \mathrm{O}$ solutions at $25^{\circ}$ ". Russian Journal of Physical Chemistry. Vol. 3, pp. 389391. 1965.

[13] Y. Marcus. "BET Modeling of Solid-Liquid Phase Diagrams of Common Ion Binary Salt Hydrate Mixtures. I. The BET Parameters". Journal of Solution Chemistry. Vol. 3, pp. 297306. 2005. 
[14] W.F. Linke, A. Seidell. Solubilities: inorganic and metal-organic compounds: a compilation of solubility data from the periodical literature. American Chemical Society, Washington DC, 1958.
[15] M.R. Ally. Líquidus curves of $\mathrm{NaNO}_{3}(\mathrm{aq})$ calculated from the modified adsorption isotherm model for Aqueous electrolytes. Monatshefte fur Chemie. Vol. 131, pp. 341344, 2000. 